\title{
Elementary Ideals in Tsemi-Rings $\operatorname{Andn}(\mathrm{A})-\mathrm{Tsemi}-\mathrm{Ring}$
}

\author{
G. Srinivasa Rao, D. Madhusudana Rao, P.Sivaprasad
}

"ABSTRACT: In this paper" we will introduce lelementary ideals, lt-elementary ideals, r-elementary ideals and elementary ideals in TSemi-rings and its properties. Also, we will discuss special type of Tsemi-rings i.e. N(I)-TSemi-ring.

Key words: Elementary ideal in T-Semi-rings, Ipotent element, I-potent I-ideal, I-divisor, $N(I)$ Tsemi-ring.

\section{INTRODUCTION}

"Derric Henry Lehmer, An American Mathematician established ternary algebraic theory. Ideal theory in ternary semigroup was introduced in 1990 by Federic Sioson. The theory of ternary and semi heaps were developed by Santiago in 1990. Shabir and Khan learnt prime ideals and prime one side ideals in semi group in the year 2008. Shabir and Bashir initiated Prime ideals in ternary semigroups.

|Succeeding that W.G.Lister read about ternary semi rings.

Revised Manuscript Received on December 16, 2019.

G. Srinivasa Rao, Associate Professor, Department of Mathematics, Tirumala Engineering College, Narasaraopeta,"A.P., India.Email:gsrinulakshmi77@gmail.com

D. Madhusudana Rao, Associate Professor, Department of Mathematics, VSR \& NVR College, Tenali, A.P. Email: dmrmaths@gmail.com”

P.Sivaprasad, Associate "Professor", Division of Mathematics,"VFSTR" Deemed to be "University, Vadlamudi,A.P., India;Email".pusaptisivaprasad@"gmail.com"
About T.K.Dutta and S.Kar initiated and considered some properties of ternary semirings which is generalization of ternary semi rings.

Dheena, P.Manvisan.S prepared a study on p-prime and small p-prime ideals in semi rings. S.Kar proved on Quasi ideals and bi-ideals in ternary semi rings. The same concept was established by Sarala, A.Anjaneyulu and D.Madhusudhanarao to ternary semi groups. In the year 2014 D.Madhusudhanarao and G.Srinivasarao investigated and read about classification of ternary semi rings and some particular elements in a ternary semirings, investigated structure of certain ideals in ternary semi rings."

\section{BASIC RESULTS}

For basic results, "we refer to the papers in the references".

\section{I.ELEMENTARY IDEALS IN TSEMI-RINGS}


Def "1.1 :An ideal I of a" Tsemi-ring T "is" said to be a $\boldsymbol{l}(\boldsymbol{l t}, \mathbf{r})$ elementary ideal is

(i) if $\mathrm{U}_{1}, \mathrm{U}_{2}, \mathrm{U}_{3}$ are "three ideals of $\mathrm{T}$ such that" $\mathrm{U}_{1} \mathrm{U}_{2} \mathrm{U}_{3} \subseteq \mathrm{I}$ and " $\mathrm{U}_{2} \nsubseteq \mathrm{I}, \mathrm{U}_{3} \nsubseteq \mathrm{I}$ ", then " $\mathrm{U}_{1} \subseteq \sqrt{\mathrm{I}}$ ". $\left(\mathrm{U}_{1} \mathrm{U}_{2} \mathrm{U}_{3} \subseteq \mathrm{I}\right.$ and $\mathrm{U}_{1} \nsubseteq \mathrm{I}, \mathrm{U}_{3} \nsubseteq \mathrm{I}$ then $\mathrm{U}_{2} \subseteq \sqrt{\mathrm{I}}, \mathrm{U}_{1} \mathrm{U}_{2} \mathrm{U}_{3} \subseteq \mathrm{I}$ and $\mathrm{U}_{1} \nsubseteq \mathrm{I}, \mathrm{U}_{2} \nsubseteq \mathrm{I}$ then $\left.\mathrm{U}_{3} \subseteq \sqrt{\mathrm{I}}\right)$. (ii) " $\sqrt{\mathrm{I}}$ is a prime ideal of T".

Ex 1.2 : "Let $T=\left\{p_{1}, p_{2}, p_{3}, p_{4}\right\}$ be a" Tsemi-ring under the ternary operation .given by

\begin{tabular}{|l|l|l|l|l|}
\hline+ & $p_{1}$ & $p_{2}$ & $p_{3}$ & $p_{4}$ \\
\hline$p_{1}$ & $p_{1}$ & $p_{2}$ & $p_{3}$ & $p_{4}$ \\
\hline$p_{2}$ & $p_{2}$ & $p_{3}$ & $p_{4}$ & $p_{1}$ \\
\hline$p_{3}$ & $p_{3}$ & $p_{4}$ & $p_{1}$ & $p_{2}$ \\
\hline$p_{4}$ & $p_{4}$ & $p_{1}$ & $p_{2}$ & $p_{3}$ \\
\hline
\end{tabular}

Define the ternary operation [] as $\left[\mathrm{U}_{1} \mathrm{U}_{2} \mathrm{U}_{3}\right]=\mathrm{U}_{1}\left(\mathrm{U}_{2} \mathrm{U}_{3}\right)=$

\begin{tabular}{|l|l|l|l|l|}
\hline$\cdot$ & $p_{1}$ & $p_{2}$ & $p_{3}$ & $p_{4}$ \\
\hline$p_{1}$ & $p_{1}$ & $p_{1}$ & $p_{1}$ & $p_{1}$ \\
\hline$p_{2}$ & $p_{1}$ & $p_{1}$ & $p_{1}$ & $p_{2}$ \\
\hline$p_{3}$ & $p_{1}$ & $p_{1}$ & $q$ & $p_{1}$ \\
\hline$p_{4}$ & $p_{1}$ & $p_{2}$ & $p_{2}$ & $p_{1}$ \\
\hline
\end{tabular}

$\left(\mathrm{U}_{1} \mathrm{U}_{2}\right) \mathrm{U}_{3}$.

Then $(T,+,[])$ is a Tsemi-ring. Let " $U_{1}=\left\{p_{1}, p_{3}\right\}, U_{2}=\left\{p_{2}, p_{2}\right\} ", U_{3}=\left\{“ p_{1}, p_{2}, p_{3}\right.$ " $\}$ and $U_{4}=\left\{\right.$ “ $p_{1}, p_{3}$, p4" $\}$. Then $\mathrm{U}_{1}, \mathrm{U}_{2}, \mathrm{U}_{3}, \mathrm{U}_{4}$ are all ideals of $\mathrm{T}$. Now $\mathrm{U}_{1} \mathrm{U}_{2} \mathrm{U}_{3} \subseteq \mathrm{U}_{1}$ and $\mathrm{U}_{1} \nsubseteq \mathrm{U}_{1}, \mathrm{U}_{3} \nsubseteq \mathrm{U}_{1}$ then $\mathrm{U}_{2} \subseteq \sqrt{\mathrm{U}_{1}}$ and $\sqrt{\mathrm{U}_{1}}$ "is a prime ideal of $\mathrm{T}$ ". Therefore $\mathrm{U}_{1}$ is a l-elementary ideal of $\mathrm{T}$.

Ex 1.3: "In the Ex 1.2, $\mathrm{U}_{1} \mathrm{U}_{2} \mathrm{U}_{3} \subseteq \mathrm{U}_{1}$ and $\mathrm{U}_{1} \nsubseteq \mathrm{U}_{1}, \mathrm{U}_{3} \nsubseteq \mathrm{U}_{1}$ then $\mathrm{U}_{2} \subseteq \sqrt{\mathrm{U}_{1}}$ and $\sqrt{\mathrm{U}_{1}}$ is a prime ideal of $\mathrm{T}$. Therefore $\mathrm{U}_{1}$ is a lt-elementary ideal of Tsemi-ring T."

Ex 1.4: "In the Ex 1.2. $U_{1} U_{2} U_{3} \subseteq U_{1}$ and $U_{1} \nsubseteq U_{1}, U_{3} \nsubseteq U_{1}$ then $U_{2} \subseteq \sqrt{U_{1}}$ and $\sqrt{U_{1}}$ is a prime ideal of $T$. Hence I is a r-elementary ideal of Tsemi-ring T."

Def 1.5 : "An ideal I of a Tsemi-ring $\mathrm{T}$ is said to be a elementary ideal provided I is a l-elementary ideal, a lt-elementary ideal and a r-elementary ideal."

Ex 1.6 : In Ex 1.2. the subset $U_{4}$ is a "elementary ideal of Tsemi-ring T."

“Th 1.7 : Let $I$ be anideal of a Tsemi-ring $T$. Then $U_{1}, U_{2}$ and $U_{3}$ are threeideals of $T$ such that $U_{1} U_{2} U_{3} \subseteq I_{\text {Iand }} \nsubseteq \Phi, W \nsubseteq I \Rightarrow U_{1} \subseteq \sqrt{ }$ Iifandonlyif $U_{1}, \quad U_{2}, \quad U_{3} \in \quad T,\left\langle U_{1}\right\rangle\left\langle U_{2}\right\rangle\left\langle U_{3}\right\rangle \subseteq I \quad$ and $\quad U_{2}$, $\mathrm{U}_{3} \notin \mathrm{I} \Rightarrow U_{I} \in \sqrt{ } \mathrm{I} . "$ 
"Th 1.8 : Let $I$ be an ideal of a Tsemi-ring $T$. Then $U_{1}, U_{2}, U_{3}$ are threeideals of $T$ such that $U_{1} U_{2} U_{3} \subseteq I$ and $U_{1} \nsubseteq I, \quad U_{3} \nsubseteq \quad I \Rightarrow U_{2} \subseteq \quad \sqrt{I} \quad$ if and only if $U_{1}, U_{2}, U_{3} \in \quad T$, $\left\langle U_{1}\right\rangle\left\langle\mathrm{U}_{2}\right\rangle\left\langle\mathrm{U}_{3}>\subseteq \mathrm{I}\right.$ and $U_{1}, \mathrm{U}_{3} \notin \mathrm{I} \Rightarrow \mathrm{U}_{2} \in \sqrt{ } \mathrm{I}$.

Th 1.9 : Let $\mathbf{I}$ be an ideal of a Tsemi-ring $T$. Then $U_{1}, U_{2}, U_{3}$ are threeideals of $T$ such that $\mathrm{U}_{1} \mathrm{U}_{2} \mathrm{U}_{3} \subseteq \quad \mathrm{I}$ and $\mathrm{U}_{1} \nsubseteq \mathrm{I}, \quad \mathrm{U}_{2} \nsubseteq \quad \mathrm{I} \Rightarrow \mathrm{U}_{3} \subseteq \quad \sqrt{\mathrm{I}} \quad$ if and only if $U_{1}, \mathrm{U}_{2}, \quad \mathrm{U}_{3} \in \quad T$, $\left\langle U_{1}>\left\langle\mathrm{U}_{2}\right\rangle\left\langle\mathrm{U}_{3}>\subseteq \mathrm{I}\right.\right.$ and $U_{1}, \mathrm{U}_{2} \notin \mathrm{I} \Rightarrow \mathrm{U}_{3} \in \sqrt{ } \mathrm{I}$."

Th1.10 : "Let $T$ be a commutative Tsemi-rings and $I$ be an ideal of $T$. Then the following conditions are equivalent.

1.I isa l-elementary $(\mathrm{lt}, \mathrm{r})$ ideal.

2. $U_{1}, U_{2}, U_{3}$ are three ideals of $T$ such that $U_{1} U_{2} U_{3} \subseteq I$ and $U_{2} \nsubseteq I, U_{3} \nsubseteq I \Rightarrow U_{1} \subseteq \sqrt{ } \mathbf{I} .\left(U_{1}, U_{2}, U_{3}\right.$ are three ideals of $T$ such that $U_{1} U_{2} U_{3} \subseteq I$ and $U_{1} \nsubseteq I, U_{3} \nsubseteq I \Rightarrow U_{2} \subseteq \sqrt{ } I$. ; $U_{1}, U_{2}, U_{3}$ are three ideals of $T$ such that $\mathrm{U}_{1} \mathrm{U}_{2} \mathrm{U}_{3} \subseteq \mathbf{I}$ and $\mathrm{U}_{1} \nsubseteq \mathrm{I},{ }^{\prime} \mathrm{U}_{3} \nsubseteq " \mathrm{I} \Rightarrow \mathbf{U}_{3} \subseteq \sqrt{ } \mathbf{I}$ ”).

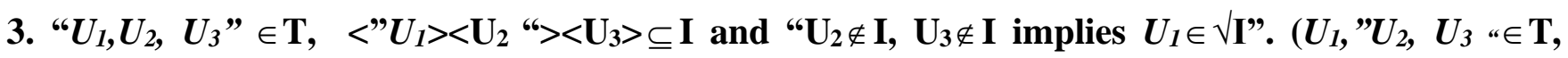
$\left\langle{ }^{\prime} U_{1}\right\rangle\left\langle\mathbf{U}_{2} "\right\rangle\left\langle\mathbf{U}_{3}\right\rangle \subseteq I$ and " $U_{1} \notin \mathbf{I}, \mathbf{U}_{3} \notin \mathbf{I}$ " implies " $\mathrm{U}_{2} \in \sqrt{ } \mathbf{I} ", U_{1}, U_{2}, " U_{3}$ " $\in \mathbf{T}, \mathrm{U}_{1} \mathrm{U}_{2} \mathrm{U}_{3} \subseteq \mathrm{I}$ and $U_{1} \notin A, U_{2} \notin I$ implies $U_{3} \in \sqrt{ }$ I.).

Th 1.11 : "Let $T$ be a commutative Tsemi-ring and $I$ be anideal of $T$. Then the following conditions are equivalent.

1) $I$ is a elementary ideal. 2) $U_{1}, U_{2}, U_{3}$ are threeideals of $T, U_{1} U_{2} U_{3} \subseteq I$ and $U_{2} \nsubseteq I, U_{3} \nsubseteq I \Rightarrow U_{1} \subseteq V_{I}$. 3) $U_{1}, U_{2}, U_{3} \in T, U_{1} U_{2} U_{3} \in I, U_{2}, U_{3} \notin I \Rightarrow U_{1} \in \sqrt{ } I$."

NOTE 1.12 : "In an arbitraryTsemi-ring a l-elementary ideal is not necessarily a r-elementary ideal." Ex 1.13 : Let $T=\left\{p_{1}, p_{2}, p_{3}\right\}$. Define binary operations addition and multiplication."in $T$ as shown in the following table."

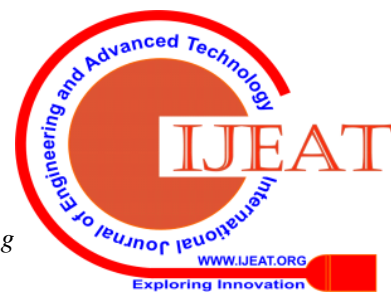


Define
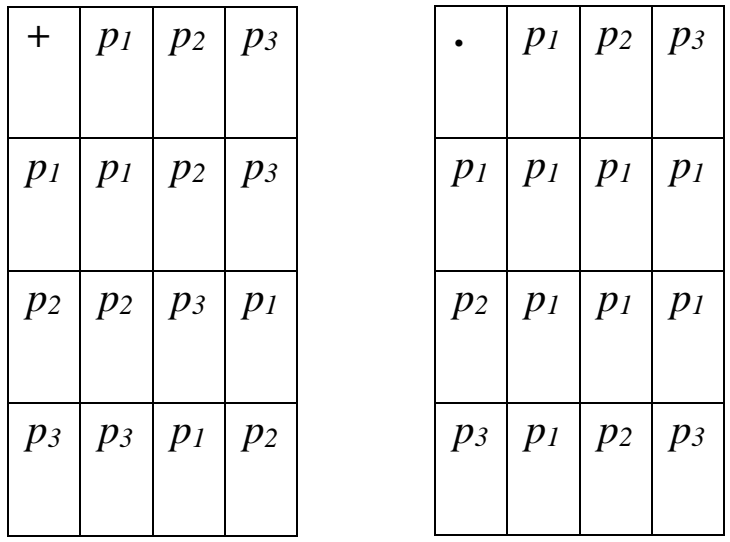

mapping from " $\mathrm{T} \times \mathrm{T} \times \mathrm{T} \rightarrow \mathrm{T}$

by $\left[\mathrm{p}_{1} \mathrm{p}_{2} \mathrm{p}_{3}\right]=\mathrm{p}_{1} \cdot \mathrm{p}_{2} \cdot \mathrm{p}_{3}$ " for all $\mathrm{p}_{1}, \mathrm{p}_{2}, \mathrm{p}_{3} \in \mathrm{T}$. "It is easy to see that $\mathrm{T}$ is a" Tsemi-ring. Now consider the ideal, $\left\langle\mathrm{p}_{1}\right\rangle=\mathrm{T}^{\mathrm{e}} \mathrm{T}^{\mathrm{e}} p_{1} \mathrm{~T}^{\mathrm{e}} \mathrm{T}^{\mathrm{e}}=\{\mathrm{p}\}$. "Let $\mathrm{abc} \subseteq\left\langle\mathrm{p}_{1}\right\rangle, \mathrm{a}, \mathrm{b} \notin\left\langle\mathrm{p}_{1}\right\rangle \Rightarrow \mathrm{b} \in \sqrt{ }\left\langle\mathrm{p}_{1}\right\rangle \Rightarrow \mathrm{b}^{\mathrm{n}} \subseteq\left\langle\mathrm{p}_{1}\right\rangle$ for some odd natural number $n \in$ N. Since $\mathrm{qrr} \subseteq\left\langle\mathrm{p}_{1}\right\rangle,{ }^{\prime} \mathrm{r} \notin\left\langle\mathrm{p}_{1}\right\rangle \Rightarrow \mathrm{p}_{2} \in \sqrt{ }\left\langle\mathrm{p}_{1}\right\rangle$. Therefore $\left\langle\mathrm{p}_{1}\right\rangle$ is 1-elementary. If $\mathrm{p}_{2} \notin\left\langle\mathrm{p}_{1}\right\rangle$ then $\mathrm{p}_{3}{ }^{\mathrm{n}} \nsubseteq<\mathrm{p}_{1}>$ for any $n \in \mathrm{N} \Rightarrow \mathrm{p}_{2} \notin \sqrt{ }<\mathrm{p}_{2}>$. Therefore $\left\langle\mathrm{p}_{1}>\right.$ is not r-elementary and lt-“elementary."

“Th 1.14 : Every ideal $I$ in a Tsemi-ring $T$ is $\mathbf{l}(\mathrm{It}, \mathrm{r})$ elementary if and only if every ideal I satisfies condition (i) of definition 1.1(1.3, 1.5)."

We now introduce the terms, a l-elementary $\mathrm{T}$ semi ring, a lt-elementary Tsemi-ring, and a relementary Tsemi-ring and anelementary Tsemi-ring.

Def 1.15 : A "T semi-ring" $\mathrm{T}$ is said to be $\boldsymbol{l}(\boldsymbol{l t}$, r)elementary provided every "ideal of $\mathrm{T}$ is a" $1(\mathrm{lt}, \mathrm{r})$ elementary "ideal" of T.

Def1.16:ATsemi-ring"Tissaidtobeelementaryprovidedeveryideal of T is a elementary ideal of T."

“Th 1.17 : Let $T$ be a $T$ semi-ring with identity and let $M$ be the unique maximal ideal of $T$. If $\sqrt{I}$ = M for some ideal of $T$, then $I$ is a elementary ideal."

NOTE 1.18: If a Tsemiring $T$ "has no identity, then the Th 1.17, is not true," even if the Tsemi-ring T has a unique maximal ideal. In Ex $1.13, \sqrt{ }<\mathrm{p}>=\mathrm{M}$ where $\mathrm{M}=\{\mathrm{p}, \mathrm{q}\}$ "is the unique maximal ideal. But $<\mathrm{p}>$ is not a elementary ideal."

"Th 1.19: If $T$ is a Tsemi-ring with identity, then for any natural number $n, M^{n}$ is elementary ideal of $T$ where $M$ is the unique maximal ideal of $T$.

NOTE 1.20: If T has no identity then Th1.17, is not true. In Ex 1.13,

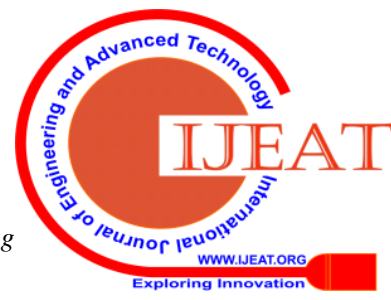


$\mathrm{M}=\{\mathrm{p}, \mathrm{q}\}$ is the unique maximal ideal, but $\mathrm{M}^{2}=\{\mathrm{p}\}$ is not elementary."

"Th 1.21: In quasi commutative Tsemi-ring $T$, an ideal $I$ of $T$ is $I(I t, r)$ elementary if and only if r(l, lt) elementary."

Cor 1.22: If " $I$ is an ideal of a quasi commutative Tsemi-ring $T$, then the following are equivalent.
1) $I$ is elementary
2) I is l-elementary
3) I is lt-elementary
4) I is r-elementary."

\section{N(I) -TSEMI-RING}

"We now introduce the terms" "I-potent element and I-potent ideal for a $\mathrm{T}$ ideal of a" $\mathrm{T}$ semiring.

Def 2.1 : "Let I be an ideal in a Tsemi-ring T. An element $\mathrm{x} \in \mathrm{T}$ is said to be I-potent $\exists$ an odd natural number $n$ such that $\mathrm{x}^{n} \in \mathrm{I}$."

Def 2.2 :"Let I be an ideal in a Tsemi-ring T. An ideal J of T is said to be I-potent ideal provided $\exists$ an odd natural number $n$ such that $\mathrm{J}^{\mathrm{n}} \subseteq \mathrm{I} \cdot "$

NOTE 2.3 : "If $I$ is an ideal of a Tsemi-ring $T$, then every element of $I$ is a I-potent element of $\mathrm{T}$ and I itself an I-potent ideal of $\mathrm{T}$.

Def 2.4 : Let I be an ideal of a Tsemi-ring T. An I-potent element $U$ is said to be a nontrivial I-potent element of $\mathrm{T}$ if $U \notin \mathrm{I}$.

NOTATION $2.5: \mathrm{N}_{\mathrm{o}}(\mathrm{I})=$ The set of all I-potent elements in $\mathrm{T}$.

$\mathrm{N}_{1}(\mathrm{I})=$ The largest ideal contained in $\mathrm{N}_{\mathrm{o}}(\mathrm{I})$.

$\mathrm{N}_{2}(\mathrm{I})=$ The union of all I-potent ideals.

Th 2.6 : If $\mathbf{I}$ is an ideal of a Tsemi-ring $\mathbf{T}$, then $I \subseteq N_{2}(I) \subseteq N_{1}(I) \subseteq N_{0}(I)$

Th 2.7 : If $I$ is an ideal in a Tsemi-ring $T$, then the following are true. 
1. $\quad \mathrm{N}_{0}(I)=A_{2} .2 \cdot N_{1}(I)$ is a semiprime ideal of $T$ containing $I .3 . N_{2}(I)=A_{4}$.

NOTE 2.8 : It is natural to ask whether $\mathrm{N}_{1}(\mathrm{I})=\mathrm{A}_{3}$. This is not true.

Ex 2.9 : In the free Tsemi-ring $\mathrm{T}$ over the alphabet $p, q, r$. For the ideal $\mathrm{I}=\mathrm{Tb}^{3} \mathrm{~T}, \mathrm{~N}_{0}(\mathrm{I})=\{\mathrm{b}\} \cup \mathrm{T}^{\mathrm{e}} \mathrm{p}^{3} \mathrm{~T}^{\mathrm{e}}$ and $\mathrm{N}_{1}(\mathrm{I})=\left\{\mathrm{p}^{3}\right\} \cup \mathrm{Tp}^{3} \mathrm{~T}=\mathrm{T}^{\mathrm{e}} \mathrm{p}^{3} \mathrm{~T}^{\mathrm{e}}$. But $\mathrm{Tp}^{3} \mathrm{~T}$ is a prime ideal, let $\mathrm{U}, \mathrm{V}, \mathrm{W}$ are three ideals of $\mathrm{S}$ such that $\mathrm{UVW} \subseteq \mathrm{T} a^{3} \mathrm{~T}$, implies all words containing $\mathrm{p}^{3} \in \mathrm{I}$ or all words containing $\mathrm{p}^{3} \in \mathrm{V}$ or all words containing $\mathrm{p}^{3} \in \mathrm{W} \Rightarrow \mathrm{I} \subseteq \mathrm{Tp}^{3} \mathrm{~T}^{\prime}$ or $\mathrm{V} \subseteq \mathrm{Tp}^{3} \mathrm{~T}$ or $\mathrm{W} \subseteq \mathrm{Tp}^{3} \mathrm{~T}$. Therefore $\mathrm{Tp}^{3} \mathrm{~T}$ isa prime ideal. We have $\mathrm{A}_{3}=\mathrm{Tp}^{3} \mathrm{~T}$, so $\mathrm{N}_{1}(\mathrm{I}) \neq \mathrm{A}_{3}$. Therefore we can remark that the inclusions in $\mathrm{A}_{3} \subseteq \mathrm{N}_{1}(\mathrm{I}) \subseteq \mathrm{N}_{0}(\mathrm{I})=\mathrm{A}_{2}$ "may be proper in an arbitraryTsemi-ring."

“Th 2.10 : If $I$ is a semipseudo symmetric ideal in a Tsemi-ring $T$, then $N_{0}(I)=N_{1}(I)=N_{2}(I)$.

Th 2.11 : For any semipseudo symmetric ideal I in a Tsemi-ring T, a nontrivial A-potent element $U(U \notin \mathrm{I})$ cannot be semisimple.

Th 2.12 : If $I$ is an ideal in a Tsemi-ring $T$, such that $N_{0}(I)=I$, then $I$ is a completely semiprime ideal and $I$ is a pseudo symmetric ideal.

Th 2.13 : If I is a semi-pseudo symmetric ideal of a semi simple Tsemi-ring the $I=N_{0}(I)$."

Def 2.14 : "Let I be an ideal in a T semi-ring T. An element $\mathrm{x} \in \mathrm{T}$ is said to be a $\boldsymbol{l}(\boldsymbol{l} \boldsymbol{t}$, $\mathbf{r}) \mathbf{I}$-divisor provided" $\exists$ two elements $y, z \in \mathrm{T} \backslash \mathrm{I}$ "such that" $x y z \in \mathrm{I}(y x z \in \mathrm{I}, y z x \in \mathrm{I})$.

Def 2.15 : "Let I be an ideal in a T semi-ring T. An element $U \in \mathrm{T}$ is said to be an I-divisor if $U$ is both a 1-I-divisor and a r-I-divisor element."

"We now introduce a l-I-divisor T ideal, lt-I-divisor T ideal, r-I-divisor T ideal and" an "Idivisor T ideal corresponding to a $\mathrm{T}$ ideal $\mathrm{I}$ in a" $\mathrm{T}$ semi-ring. 
Def 2."16 :Let I be an ideal in a" Tsemi-ring "T. An" ideal $\mathrm{J}$ in "T is said to be a" $\boldsymbol{l}(\boldsymbol{l t}$, $\mathbf{r}) \mathbf{I}$-divisor “ideal provided every element of $\mathrm{J}$ is a" 1 (lt, r) "I-divisor element".

Def 2.17 : Let I "be an ideal in a" Tsemi-ring "T. An ideal" $\mathrm{J}$ "in $\mathrm{T}$ is said to be an $\mathrm{I}$-divisor idealprovided if it is both a" 1-I-“divisor ideal and a r-I-divisor ideal of a T"semi-ring T.

"NOTATION $2.18: \mathrm{R}_{l}(\mathrm{I})=$ The union of all 1-I-divisor ideals in T".

$$
\begin{aligned}
& \mathrm{R}_{\mathrm{r}}(\mathrm{I})=\text { The union of all r-I-divisor ideals in } \mathrm{T} \text {. } \\
& \mathrm{R}_{\mathrm{m}}(\mathrm{I})=\text { The union of all lt- or medial I-divisor ideals in } \mathrm{T} \text {. } \\
& \mathrm{R}(\mathrm{I})=\mathrm{R}_{l}(\mathrm{I}) \cap \mathrm{R}_{\mathrm{m}}(\mathrm{I}) \cap \mathrm{R}_{\mathrm{r}}(\mathrm{I}) \text {. We call } \mathrm{R}(\mathrm{I}) \text {, the divisor radical of } \mathrm{T} \text {." }
\end{aligned}
$$

Th 2.19: "If Iis any ideal of a Tsemi-ring $T$, then $\mathbf{N}_{1}(I) \subseteq R(I)$.

Th 2.20 : If $I$ is an ideal in a Tsemi-ring $T$, then $R(I)$ is the union of all I-divisor ideals in $T$.

COROLLARY 2.21 : If $A$ is a pseudo symmetric ideal in a Tsemi-ring $T$, then $R(I)$ is the set of all I-divisor elements in T."

"We now introduce the notion of" N(I)-Tsemi-ring.

"Def2.22:Let I be an ideal in a T semi-ringT. T is said to be a $N(I)-T$ semi-ring provided every I-divisor is I-potent.

NOTATION 2.23 : Let $\mathrm{T}$ be a Tsemi-ring with zero. If $\mathrm{I}=\{0\}$, then we write $\mathrm{R}$ for $\mathrm{R}(\mathrm{I})$ and $\mathrm{N}$ for $\mathrm{N}_{0}(\mathrm{I})$ and N- Tsemi-ring for N(I)-Tsemi-ring.

Th $2.24:$ If $T$ is an $N(I)$-Tsemi-ring, then $R(I)=N_{1}(I)$.

Th 2.25 : Let I be a semipseudo symmetric ideal in a Tsemi-ring T. Then $T$ is an N(I)-Tsemi-ring if and only if $R(I)=N_{0}(I) . "$

Cor 2.26 : "Let I be a pseudo symmetric ideal in a Tsemi-ring $T$. Then $T$ is an N(I)-Tsemi-ring if and only if $R(I)=N_{0}(I)$.

Cor 2.27 : Let $\mathrm{T}$ be a semi-ringwith 0 and $<0>$ is a pseudo symmetric ideal. Then $\mathrm{R}=\mathrm{N}$ if and only if $\mathrm{T}$ is an $\mathrm{N}$-Tsemi-ring. 
Th 2.28: If $M$ is a maximal ideal in a Tsemi-ring $T$ containing a pseudo symmetric ideal $I$, then $M$ contains all A-potent elements in $\mathrm{T}$ or $\mathrm{T} \backslash \mathrm{M}$ is singleton which is I-potent.

Cor2.29 : If $\mathrm{M}$ is a nontrivial maximal ideal in a Tsemi-ring $\mathrm{T}$ containing $a$ pseudo symmetric ideal I. Then $\mathrm{N}_{0}(\mathrm{I}) \subseteq \mathrm{M}$.

Cor 2.30 : If $M$ is a maximal ideal in a semisimple Tsemi-ring $T$ containing $a$ semipseudo symmetric ideal I. Then $\mathrm{N}_{0}(\mathrm{I}) \subseteq M . "$

\section{Conclusion :}

"We are expressing thankful to every reviewer and give your valuable suggestions."

\section{REFERENCE}

1. “Anjaneyulu A (1980), Pimary ideals in semigroups Semigroup Form. 20:129-144.

2. Arif Kaya and Satyanarayana M". Semi-rings "satisfying properties of distributive type, Proceeding of the American Mathematical Society, Volume 82, Number 3, July 1981.

3. "Chinaram, R., A note on quasi-ideal in" semi-rings, “Int. Math. Forum, 3 (2008), 1253-1259”.

4. "Daddi. V. R and Pawar". V. S. "Ideal Theory in Commutative" T “A”-semi-rings,

5. "International Mathematical Forum, Vol. 7, 2012, no. 42, $2085-2091 ”$

6. "Dixit" YN, "Dewan. S" (1995). "A note on quasi and bi-ideals in ternary semigroups. Int. J. Math. Sci. $18 ”: 501-508$

7. “D. Madhusudana Rao and" G "Srinivasa" Rao, “A Study On Ternary Semi"-rings, IJMA- 5(12), Dec. 2014.

8. "Dutta, T.K. and Kar, S., On regular Ternary" semirings, "Advances in Algebra, Proceedings of the ICM
Satellite Conference in Algebra and Related Topics, World" Scientific, "New Jersey, 2003, 343-355".

9. "Dutta, T.K. and Kar, S., A note on regular Ternary" semi-rings, "Kyung-pook Math. ., 46 (2006), 357-365”.

10. "Iampan A (2007). Lateral ideals of Ternary semigroups - Yxpaihckkhii Mate. Math. Torn. 4(4):525-534”.

11. "Jonathan S. Golan". Semi-rings "and Affine Equations over Them: Theory and Applications, Kluwer Academic".

12. "Kerner R(2000). $T$ Algebraic Structures and their Applications Univ.P\&M, Qurie-print, Paris, Arixiv Math. 4:525-534”.

13. "Kar, S., On quasi-ideals and bi-ideals in Ternary" semi-rings, "Int. J. Math. Math. Sc., 18 (2005),3015$3023 \%$

14. "Lehmer" DH (1932). "A ternary analogue of abelian groups, Amer. J.Math. 59":329-338.

15. "Lister, W.G., Ternary rings, Trans Amer. 
Math.Soc., 154 (1971), 37\{55\}".

16. "Los J (1955). On the extending of model I.

Fundamenta Mathematicae, 42: 38-54”.

17. "Madhusudhana Rao. D., Elementary Ideals in QuasiCommutative Ternary Semigroups International Research Journal of Pure Algebra - 3(7), 2013, 254258.

18. MadhusudhanaRao. D. and SrinivasaRao.G., Structure of Certain Ideals in Ternary” semi-rings, “International Journal of Innovative Science and Modern Engineering(IJISME)-Volume-3, Issue-2, January, $2015 "$

19. "Shabir M, Bashir S (2009). Prime ideals in ternary semigroups, Asian Eur.J. Math. 2:139-152”.

20. "Santiago M. L (1990). Regular ternary semigroups. Bull. Calcutta Math.Soc. 82:67-71”.

21. "Shabir M, Khan A (2008).Fuzzy filters in ordered semigroups.Lobachevskii J. Math. 29:82-89”.

22. "Sioson FM (1965). Ideal theory in ternary semigroups, Math”.Soc.

23. Whan, "J. and Dudek, W.A., Fuzzy h-ideals of hemirings, Inform. Sci., 177 (2007), 876- 886”. 